\title{
Autonomous observatories for the Antarctic plateau
}

\author{
J. S. Lawrence ${ }^{1}$, M. C. B. Ashley ${ }^{2}$, and J. W. V. Storey ${ }^{2}$ \\ ${ }^{1}$ Australian Astronomical Observatory, North Ryde, \\ NSW 2113, Australia, \\ email: j1@aao.gov.au \\ ${ }^{2}$ School of Physics, University of New South Wales, \\ Sydney, NSW 2052, Australia \\ email: m.ashley@unsw.edu.au, j.storey@unsw.edu.au
}

\begin{abstract}
Antarctic astronomical site-testing has been conducted using autonomous selfpowered observatories for more than a decade (the AASTO at South Pole, the AASTINO at Dome C, and PLATO at Dome A/Dome F). More recently autonomous (PLATO) observatories have been developed and deployed to support small-scale scientific instruments, such as HEAT, a $0.6 \mathrm{~m}$ aperture terahertz telescope at Ridge A, and AST3, a $0.5 \mathrm{~m}$ optical telescope array at Dome A. This paper reviews the evolution of autonomous Antarctic astronomical observatories, and discusses the requirements and implications for observatories that will be needed for future larger-scale facilities.
\end{abstract}

Keywords. Antarctic astronomy, site testing, autonomous observatory

\section{Introduction}

Conditions on the Antarctic plateau are very promising for astronomy. The high altitude, low surface winds, and lack of a tropospheric jet stream lead to a beneficial turbulence profile. The cold and dry atmosphere leads to low thermal emission and high throughput. The cloud free conditions and the high site latitudes are ideal for highcadence imaging programs. The initial verification of these conditions, and of the feasibility of conducting observations that take advantage of these conditions, has required the development of astronomical observatories that could operate in extreme environmental conditions with a high level of automation.

Potential sites for astronomy are at permanently manned stations such as the US Amundsen Scott South Pole station operated since the late 1950s, and the French/Italian Dome C station operated year round since 2005; mostly summer-only stations such as the Japanese base at Dome F operated since 1995, the Chinese Kunlun station at Dome A since 2007, and the Russian Vostok station operated since 1957; or field stations such as Ridge A operated since 2012.

Manned observatories at permanently-manned and summer-only Antarctic plateau stations have been vital for the collection of site testing and science data. Facilities at the South Pole have supported a range of telescopes: SPT, BICEP, Viper-ACBAR, DASI, AST/RO and SPIREX. At Dome C, facilities have supported the AstroConcordia program, A-STEP, COCHISE and IRAIT.

Collection of winter-time data from summer-only stations or from field locations has required the development of fully autonomous self-powered observatories, which have now been deployed to a number of sites on the high plateau. These observatories have significantly progressed in capability and reliability over the last fifteen years. 


\section{Autonomous Observatory Requirements}

The primary requirements for a remote self-powered autonomous observatory for astronomy on the Antarctic plateau include:

(a) The observatory must provide electrical power to instruments. The total amount of power depends on the type and range of instrumentation but is usually in the range 10-100 W for a single experiment and 1-3 kW for an instrument suite or a moderate size science experiment.

(b) The observatory must provide heat to keep instrument components above ambient temperature. The total heat required depends on the instrument elements and the instrument size but generally the heat required is of similar magnitude to the electrical power required.

(c) The observatory must provide an autonomous and reliable control system. This system includes both the power distribution system which must have multiple redundancies and the computer control system.

(d) The observatory must provide an autonomous and reliable communications system. Communication is required for observatory housekeeping and control and for data and control of instrumentation.

(e) The observatory must comply with appropriate logistical constraints. Constraints are imposed by the scale of the observatory and its intended location and are primarily dictated by the method of transportation (e.g., Twin Otter, LC130 aircraft, ice sled, helicopter, icebreaker, etc).

\section{AASTO: South Pole}

The Automated Antarctic Site Testing Observatory (AASTO; Fig. 1) was deployed to South Pole in Jan 1997 (Storey et al. 1996). This was a collaboration between Australian and US institutes under the umbrella of the Center for Astrophysical Research in Antarctica (CARA) and the Joint Australian Centre for Astrophysical Research in Antarctica (JACARA). The AASTO was fabricated from a single fibreglass module based on a modified Automated Geophysical Observatory (AGO; Dudeney et al. 1998). Similar to the AGO program, the AASTO was designed to be transported to remote locations on the plateau via ski-equipped LC130 aircraft. The AASTO was initially powered via a Thermo-Electic Generator (TEG) that produced $\sim 50 \mathrm{~W}$ electrical power and $\sim 2 \mathrm{~kW}$ of heat. As was also the case in the AGO program, the TEG proved unreliable for unmanned operation and the AASTO was switched to South Pole station power.

The AAO control system consisted of a series of PC104-based computers communicating with instruments via 1-wire-bus and/or a serial ports (RS232/485). Communication was via the South Pole station network linked to a series of inclined geosynchronous satellites.

The AASTO was initially used to support a suite of site-testing instruments, including a mid-infrared sky monitor (MISM; Chamberlain et al. 2000), a near-infrared sky monitor (NISM; Lawrence et al. 2002), a sonic radar (SODAR; Travouillon et al. 2003), a submillimetre tipping radiometer (SUMMIT; Calisse et al. 2004), and a telescope fibrecoupled to an optical spectrograph (AFOS; Dempsey et al. 2004). It was later also used as a platform for science experiments, such as the Vulcan South Pole transit search experiment. The AASTO was decommissioned in 2005. 


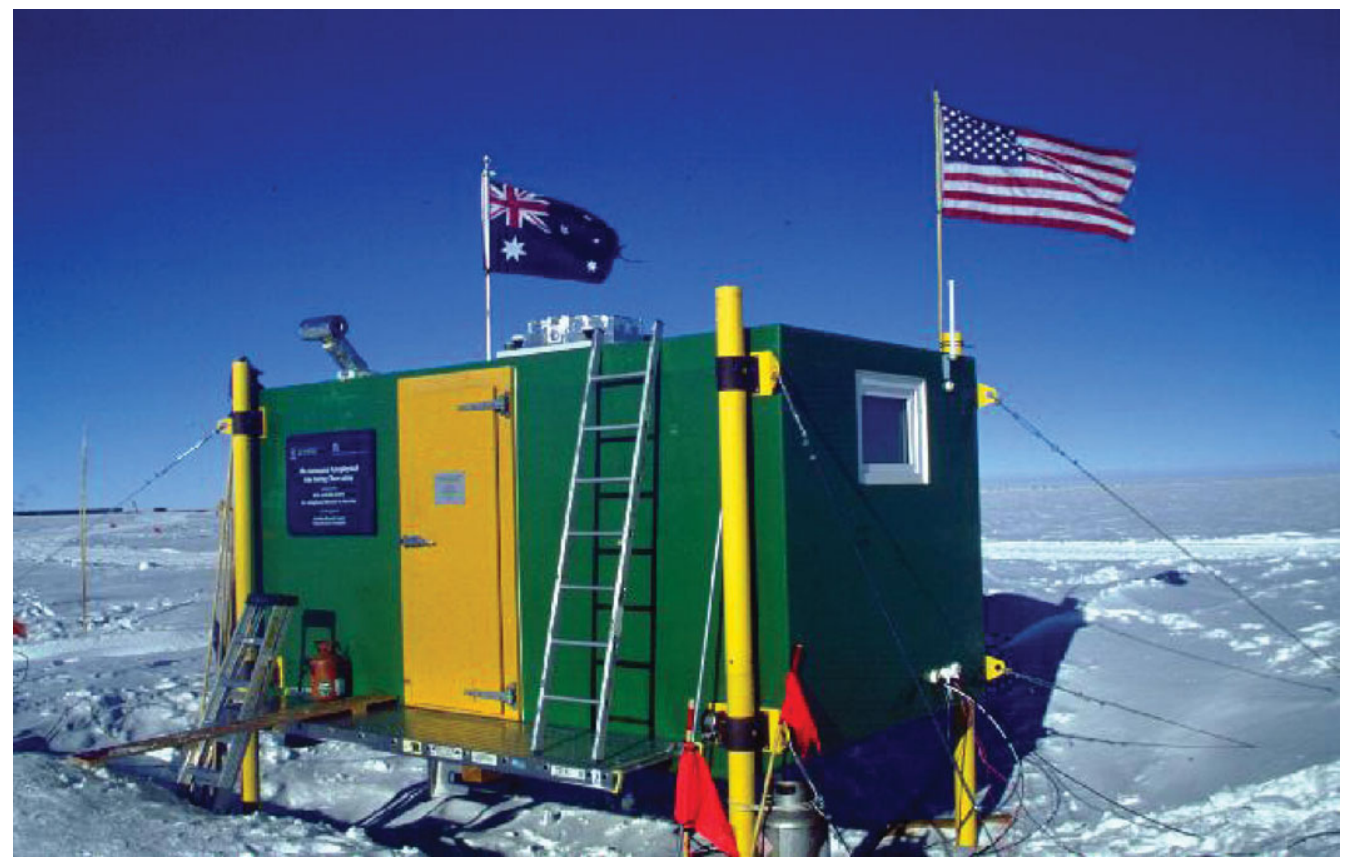

Figure 1. The AASTO at the South Pole from 1997.

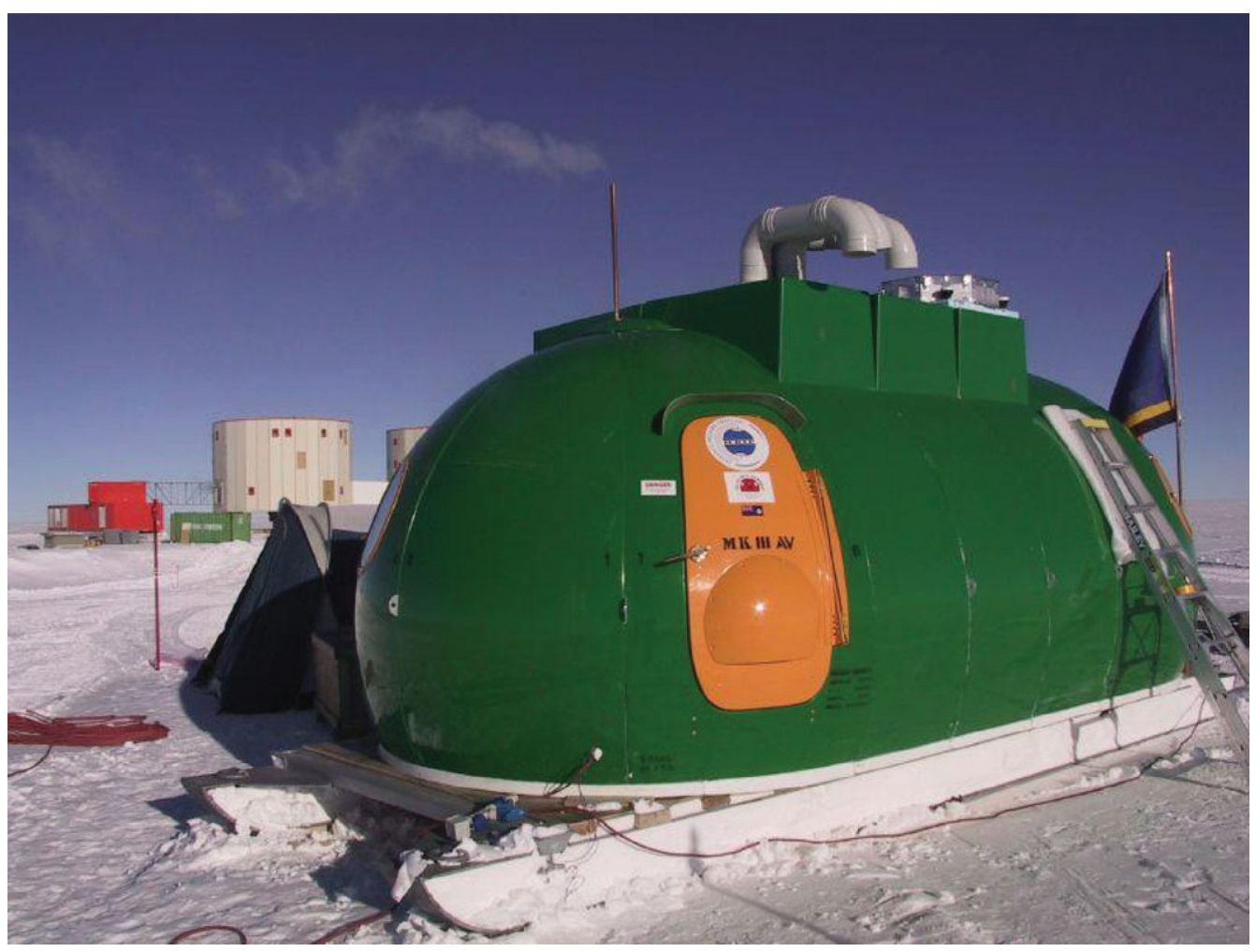

Figure 2. The AASTINO at Dome C in 2003. 


\section{AASTINO: Dome C}

The Automated Antarctic Site Testing INternational Observatory (AASTINO; Fig. 2) was deployed to the Italian French Dome C station in Jan 2003 (Lawrence et al. 2005). It was designed to support a series of site-testing instruments during the winter months prior to the station opening for full winter manned operation. The AASTINO was a collaboration between the University of New South Wales and the Australian, French and Italian national Antarctic agencies (AAD, IPEV and PNRA). Institutes from Australia, France, Italy and the US contributed to the instrument suite.

The AASTINO was constructed from an outer fibreglass casing with thick internal sprayfoam insulation. The entire module was assembled from a series of 16 panels onsite. Transport for the AASTINO was via icebreaker (from Tasmania to the Antarctic coastal station Dumont Durville) then via overland tractor traverse to Dome C.

Power and heat for the AASTINO were provided via two Stirling cycle engines running on JetA1 aviation fuel. They provided $\sim 400 \mathrm{~W}$ peak electrical power and several $\mathrm{kW}$ of heat. This was augmented with a solar panel bank that provided $400 \mathrm{~W}$ during the summer months.

The AASTINO used a similar (though upgraded) 1-wire communications bus, control architecture and PC104 computer system to the AASTO. For communication the AASTINO used the Iridium satellite system.

The instrument suite for AASTINO included a multi-aperture scintillation sensor (MASS; Lawrence et al. 2004), the SUMMIT sub-millimetre tipping radiometer (Calisse et al. 2004), and an acoustic radar (SODAR; Travouillon et al. 2003).

The AASTINO ran for $\sim 6$ months in both 2003 and 2004, providing valuable data on the atmospheric qualities of the Dome $\mathrm{C}$ site well into the winter months of these years. The observatory was decommissioned in 2005, when the Concordia station was opened for year-round operation.

\section{PLATO: Dome A}

The PLATeau Observatory (PLATO; Fig. 3) was deployed to Dome A in Jan 2008 via an overland traverse conducted by the Polar Research Institute of China (PRIC). PLATO (Lawrence et al. 2009) was designed and constructed by UNSW in consultation with a number of teams from China and the US who provided site testing instruments (Yang et al. 2009).

PLATO departs from earlier observatories in a number of design aspects. A bank of 6 single cylinder diesel engines are used as the primary power source for PLATO, with by default, a single engine running at any one time. The engines, fuel (4000 litres of Jet A1), and power control electronics, are installed inside a heavily insulated customised 10 foot steel shipping container, "the engine module". A second "instrument" module (of similar construction) is installed $\sim 50$ metres away, near a solar-panel array. The PLATO instrument module houses the computer control system, instrument control computers, and a series of site-testing instruments mounted through the module wall or ceiling. The PLATO control system features many improvements over earlier observatories, in particular, it uses a CAN-bus network for subsystem control.

PLATO has collected data during the winter months at Dome A from a number of instruments, including: the CSTAR telescope array (Zhou et al. 2010), the Snodar ground layer turbulence profiler (Bonner et al. 2010), a sub-millimetre Fourier Transform spectrometer, the Gattini sky camera, the Pre-HEAT terahertz telescope (Yang et al. 2010), and the Nigel fibre optical spectrograph (Sims et al. 2012). 


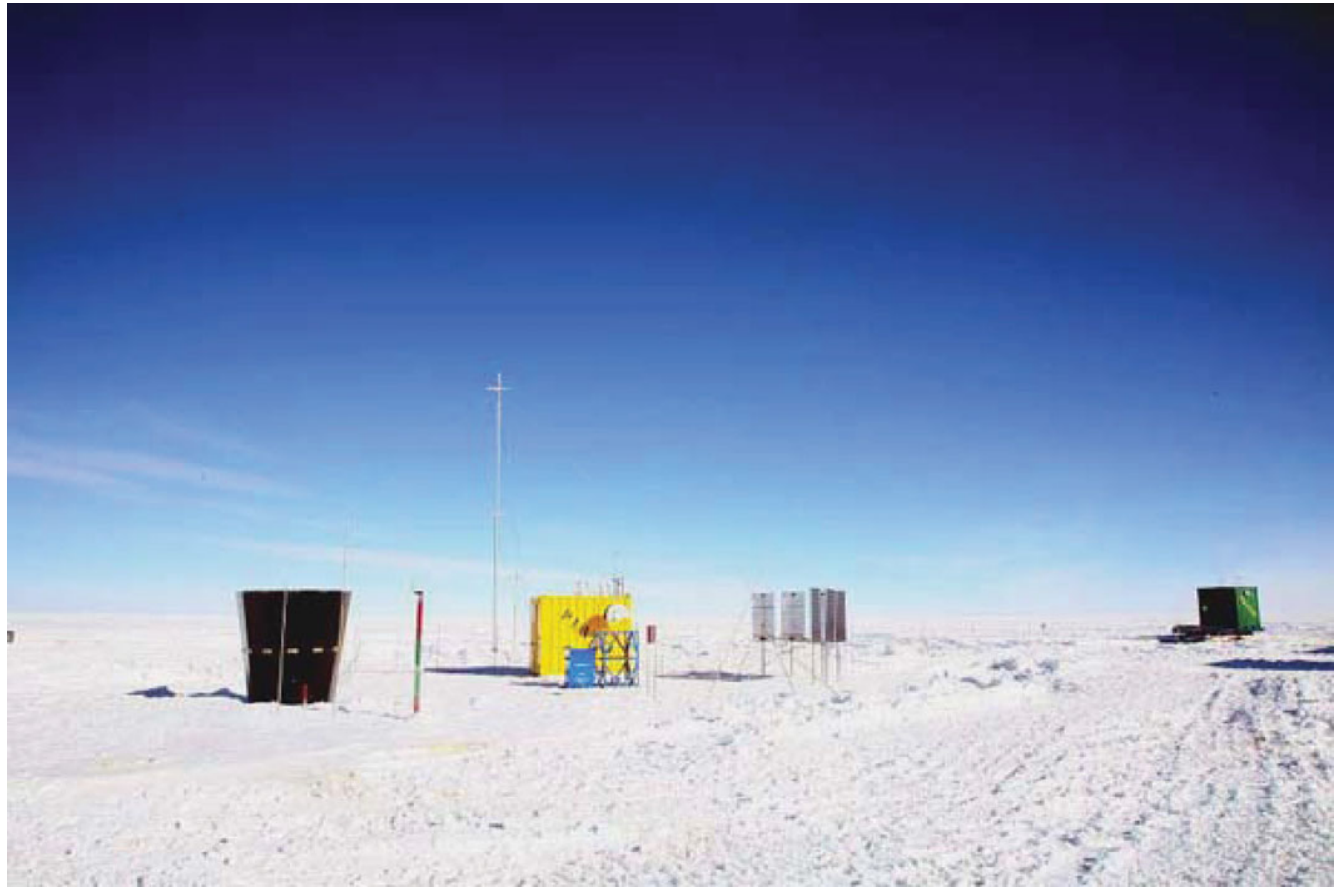

Figure 3. PLATO installed at Dome A in 2008.

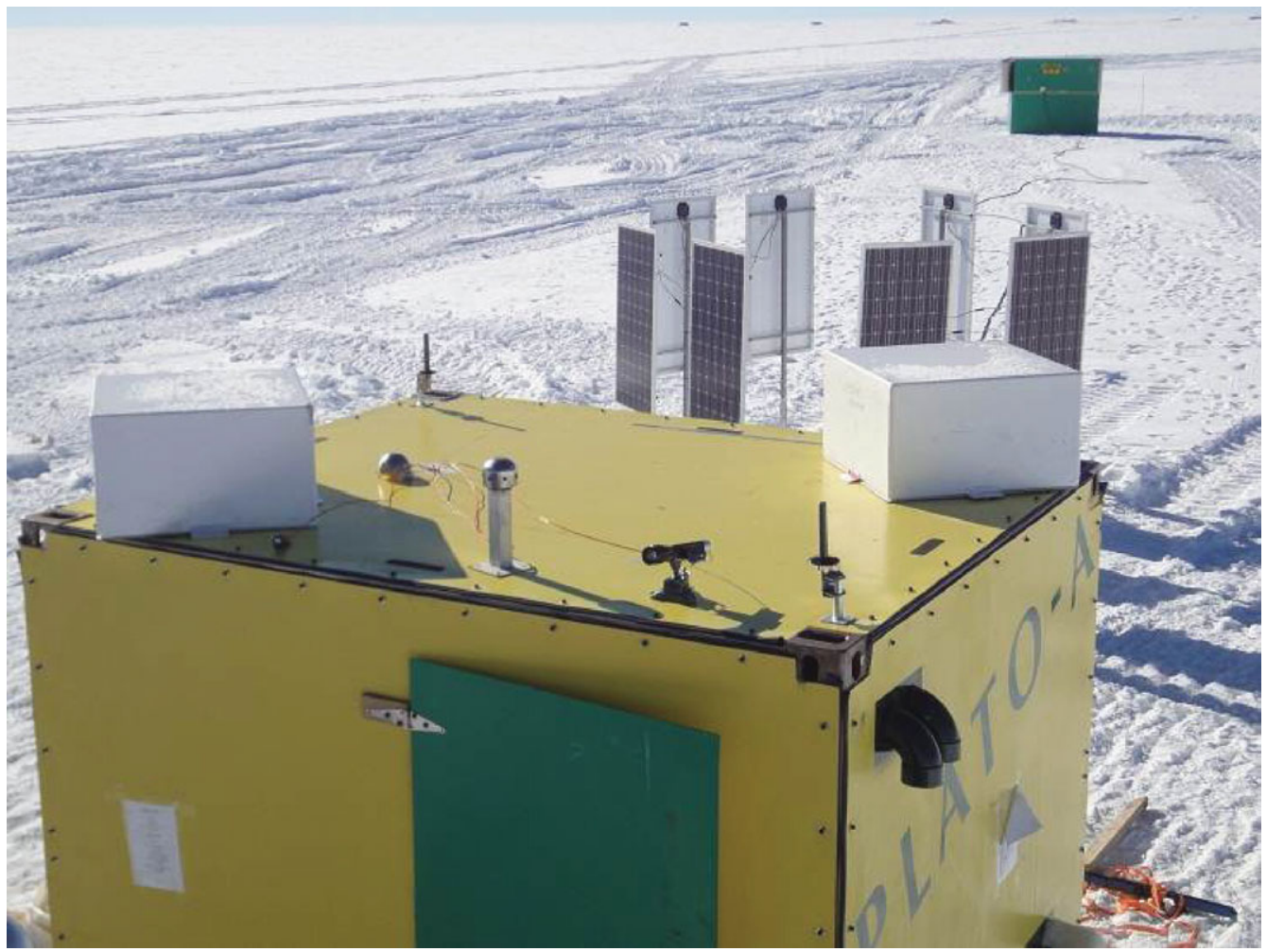

Figure 4. PLATO-A installed at Dome A in 2012. 
The PLATO observatory has been extremely successful: running autonomously for more than 200 days in its first winter of 2008, and running continuously (with yearly servicing missions) for more than 1230 days since 2009.

\section{PLATO-A: Dome A}

In Jan 2012, the PLATO-A (Fig. 4) observatory was deployed to Dome A, just $250 \mathrm{~m}$ from the original PLATO. The sole purpose of PLATO-A is to provide a power, control, and communications platform for the AST3 telescope array (Yuan \& Su 2012), developed by a consortium of Chinese institutes.

The PLATO-A observatory is similar in most aspects to the original PLATO observatory. It is powered by a bank of five single-cylinder diesel engines with a solar panel array, capable of providing up to $3 \mathrm{~kW}$ of heat and peaks of $3 \mathrm{~kW}$ of electrical power. It comprises two separate modules, one for instrument control and one for power generation (though these are fibreglass construction rather than steel as in the original PLATO). Modifications have also been made to the power system control architecture to enhance reliability and operability.

The first telescope in the AST3 array was installed with PLATO-A in Jan 2012. At time of writing, the PLATO-A observatory is still functional though a power supply problem caused the telescope operation to cease. A servicing mission for the observatory will coincide with the deployment of the second AST3 telescope in Jan 2013.

\section{PLATO-F: Dome F}

With a view to collecting data on the site qualities of the Dome F location, Japanese and Australian institutes and polar research agencies collaborated on the development of the PLATO-F observatory. PLATO-F (Fig. 5) was deployed to Dome F in Jan 2011.

The PLATO-F observatory is similar to the PLATO-A (second generation) observatory at Dome A. The power generation system for PLATO-F consists of a bank of 5 single cylinder diesel engines plus a solar panel array. The observatory comprises two modules (one for the engines and one for the instruments and control system). These modules were designed with a focus on minimising weight so that the observatory could be assembled from individual sections at the Japanese coastal station after transfer from the icebreaker via helicopter.

The instrument suite for PLATO-F comprises a Snodar ground-layer turbulence profiler, the Twincam optical telescope experiement, and a metrology tower. PLATO-F ran unattended for more than half a year in 2011. The next servicing mission is planned for Jan 2013.

\section{PLATO-R: Ridge A}

The site at Ridge A was identified as likely to experience lower water vapour content than Dome A (Saunders et al. 2009), motivating the development of the PLATO-R observatory (Fig. 6). PLATO-R was designed specifically to provide power and control for the HEAT terahertz telescope. Both were deployed to Ridge A in Jan 2012.

Significant constraints were placed on the PLATO-R design because the Ridge A location had no infrastructure as it had previously not been visited. Transport was viable only via ski-equipped Twin Otter aircraft. This meant that all components of PLATO-R had to fit within the limited cargo bay doors and load capacity for this aircraft. The observatory architecture for PLATO-R was similar to previous PLATO versions, i.e., with 


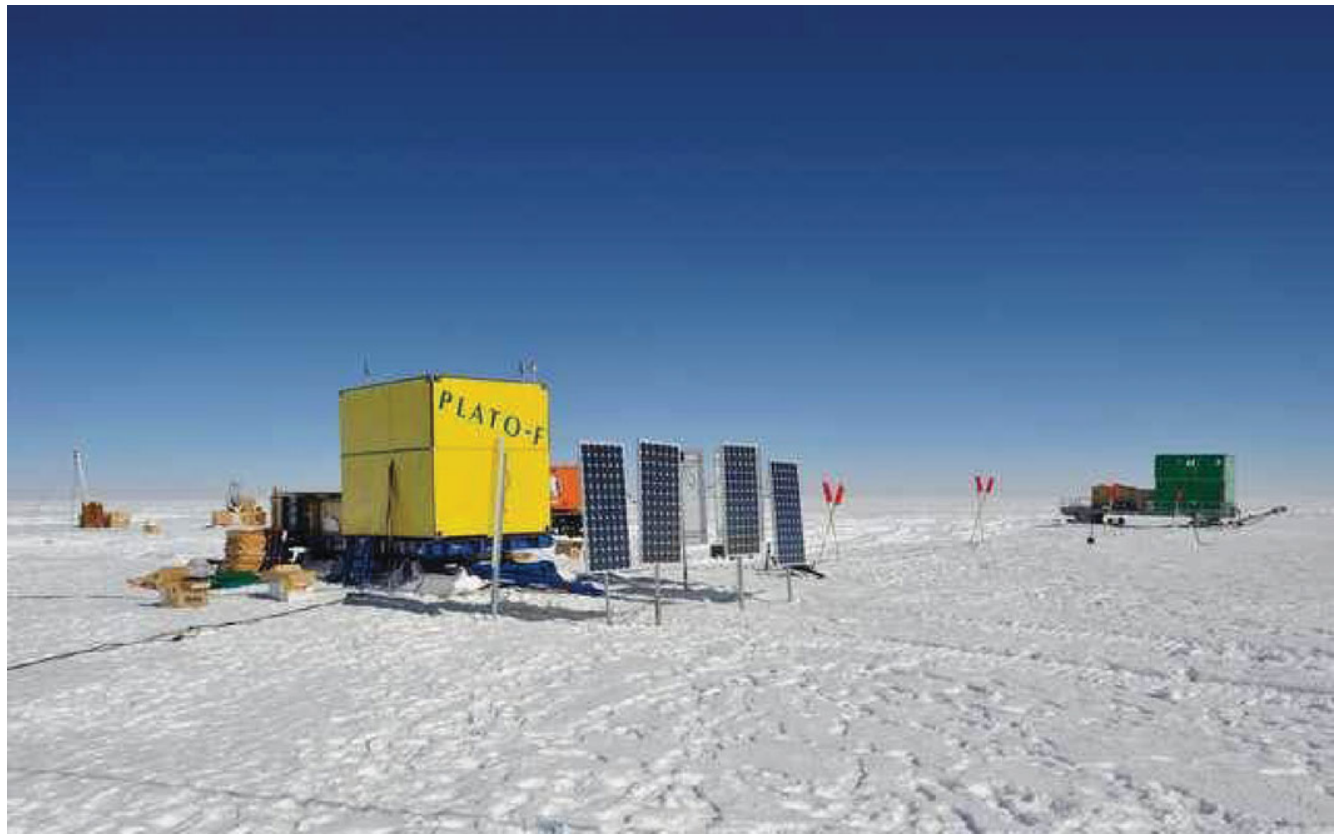

Figure 5. PLATO-F installed at Dome F in 2011.

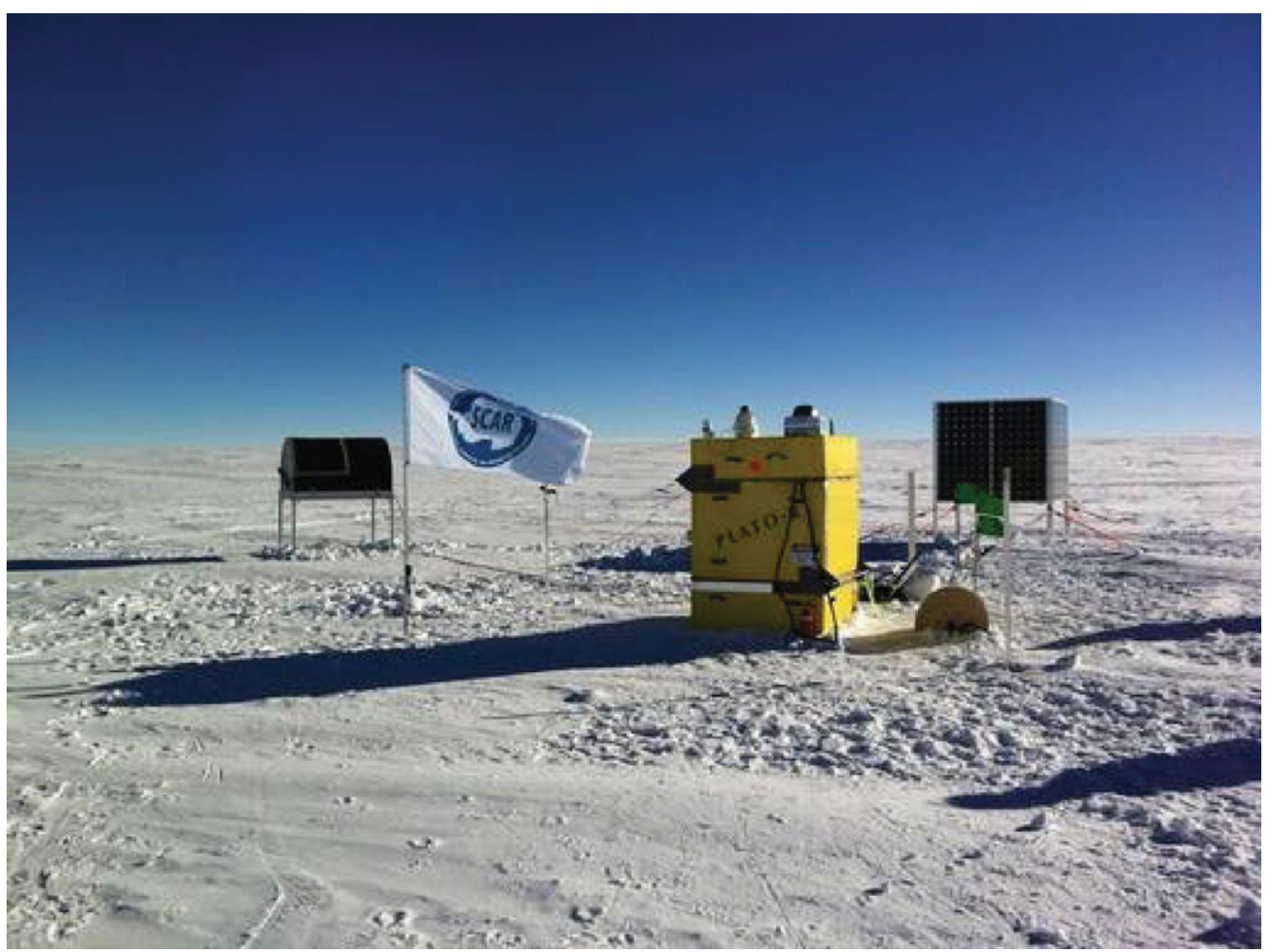

Figure 6. PLATO-R installed at Ridge A in 2012. 
separate engine and instrument modules and external solar panels. For PLATO-R these modules are both relatively small. Both are constructed from fibreglass. The engine module houses two diesel engines, the instrument module houses the iridium satellite system and observatory supervisor computer and electronics.

PLATO-R ran for 127 days in 2012, limited by the available Jet-A1 fuel supply. The next servicing mission is planned for the Austral summer of 12/13.

\section{Conclusion}

Autonomous self-powered observatories have been crucial for collecting data on the site conditions at unmanned Antarctic plateau locations. These observatories have progressed in capability and reliability over the last fifteen years. The field has evolved such that unmanned observatories now being deployed are powering instruments targeting astronomical science rather than atmospheric site qualification. Additionally, experiments are now possible in new locations that have no existing infrastructure.

Current generation observatories are scalable, though not indefinitely so. Future telescopes of diameter greater than $\sim 2$ metres will likely need to be deployed at permanently manned locations (so the design costs are not prohibitively expensive). However, many of the subsystems used in self-powered observatories (such as power control and communications systems) will be highly applicable to such systems. It is likely to be some time before permanently manned stations are operational at all high plateau sites and thus there are many years for which self-powered autonomous observatories will be essential for the collection of wintertime data.

\section{References}

Bonner, C. S., Ashley, M. C. B., Cui, X., Feng, L., Gong, X., Lawrence, J. S., Luong-Van, D. M., Shang, Z., Storey, J. W. V., Wang, L., Yang, H., Yang, J., \& Zhou, X. 2009, P.A.S.P, 122, 1122

Calisse, P. G., Ashley, M. C. B., Burton, M. G., Phillips, M. A, Storey, J. W. V., Radford, S. J. E. \& Peterson, J. B. 2004. P.A.S.A, 21, 256

Chamberlain, M. A., Ashley, M. C. B., Burton, M. G., Phillips, A., Storey, J. W. V., \& Harper, D. A. 2000, AP.J., 535, 501

Dempsey, J. T., Storey, J. W. V., Ashley, M. C. B., Burton, M. G., Calisse, P. G. \& Jarnyk, M. 2004. Proc. SPIE, 5492, 811

Dudeney, J. R., Kressman, R. I., \& Rodger, A. S. 1998, Antarct. Sci., 10, 192

Lawrence, J. S., Ashley, M. C. B., Burton, M. G., Calisse, P. G., Everett, J. R., Pernic, R. J., Phillips, A., \& Storey, J. W. V. 2002, P.A.S.A, 19, 328

Lawrence, J. S., Ashley, M. C. B., Kenyon, S., Storey, J. W. V., Tokovinin, A., Lloyd, J. P., \& Swain, M. 2004, Proc. SPIE 5489, 174

Lawrence, J. S., Ashley, M. C. B., \& Storey, J. W. V. 2005, Aus. J. Electic. Electron. Eng., 2, 1

Lawrence, J. S., Ashley, M. C. B., Hengst, S., Luong-Van, D. M., Storey, J. W. V., Yang, H., Zhou, X., \& Zhu, Z. 2009, Rev. Sci. Instrum., 80, 064501

Saunders, W., Lawrence, J. S., Storey, J. W. V., Ashley, M. C. B., Kato, S., Minnis, P., Winker, D. M., \& Liu, G., Kulesa C. 2009, P.A.S.P, 121, 976

Sims, G., Ashley, M. C. B., Cui, X,. Everett, J. R., Feng, L., Gong, X., Hengst, S., Hu, Z., Kulesa, C., Lawrence, J. S., Luong-Van, D. M., Ricaud, P., Shang, Z., Storey, J. W. V., Wang, L., Yang, H., Yang, J., Zhou, X., \& Zhu, Z. 2012, P.A.S.P, 124, 74

Storey, J. W. V., Ashley, M. C. B., \& Burton, M. G. 1996, P.A.S.A, 13, 35

Travouillon, T., Ashley, M. C. B., Burton, M. G., Storey, J. W. V., \& Loewenstein, R. F. 2003, A $\&$ A , 400, 1163 
Yang, J., Allen, G., Ashley, M. C. B., Bonner, C. S., Bradley, S., Cui, X., Everett, J. R., Feng, L., Gong, X., Hengst, S., Hu, J., Jiang, Z., Kulesa, C. A., Lawrence, J. S., Li, Y., Luong-Van, D., McCaughrean, M. J., Moore, A. M., Pennypacker, C., Qin, W., Riddle, R., Shang, Z., Storey, J. W. V., Sun, B., Suntzeff, N., Tothill, N. F. H., Travouillon, T., Walker, C. K., Wang, L., Yan, J., Yang, J., York, D., Yuan, X., Zhang, X., Zhang, Z., Zhou, X., \& Zhu, Z. 2009, P.A.S.P., 121, 174

Yang, H., Kulesa, C. A., Walker, C. K., Tothill, N. F. H., Yang, J., Ashley, M. C. B., Cui, X., Feng, L., Lawrence, J. S., Luong-Van, D. M., Storey, J. W. V., Wang, L., Zhou, X., \& Zhu, Z. 2010, P.A.S.P, 122, 490

Yuan, X. \& Su, D. 2012, MNRAS, 424, 23

Zou, H., Zhou, X., Jiang, Z., Ashley, M. C. B., Cui, X., Feng, L., Gong, X., Hu, J., Kulesa, C. A., Lawrence, J. S., Li, G., Luong-Van, D. M., Ma, J., Moore, A. M., Pennypacker, C. R., Qin, W., Shang, Z., Storey, J. W. V., Sun, B., Travouillon, T., Walker, C K., Wang, J., Wang, L., Wu, J., Wu, Z., Xia, L., Yan, J., Yang, J., Yang, H., Yao, Y., Yuan, X., York, D. G., Zhang, Z., \& Zhu, Z. 2010, A.J., 140, 602 